\title{
Qualitative research in medicine and healthcare: Is it subjective, unscientific or second class science?
}

\author{
Sayeeda Rahman ${ }^{\text {, }}$ Md. Anwarul Azim Majumder ${ }^{2}$ \\ ${ }^{1,2}$ Lecturer, Department of Clinical Sciences, School of Medical Sciences, University of Bradford, West Yorkshire, Bradford, UK.
}

Traditionally, qualitative research is not widely used in medicine and healthcare research and is usually dismissed as 'less scientific, less valid, and less objective' and 'a second-class approach'.' A recent study reported that the proportion of qualitative research in medical journals remains low despite an increase in the proportion of papers. $^{2}$ The study found a $2.9 \%$ absolute increase and 3.4-fold relative increase in qualitative research publications occurred over a 10 -year period $\left(1.2 \%\right.$ in 1998 vs. $4.1 \%$ in 2007). ${ }^{2}$ Health service research is generally quantitative and based on biomedical traditions and experimental methods. One of the important reasons for the low use of qualitative research may be that researchers are unfamiliar with this method and are unsure about how it relates to their interests or to their field. ${ }^{3}$ This generates considerable 'confusion' and 'controversy' about how qualitative research can address 'clinical' or 'bio-psychosocial' aspects of medicine and healthcare. ${ }^{3}$ Some researchers suggested that the scope of qualitative research in medicine and healthcare is much narrower and ignore traditional clinical concerns. ${ }^{4.5}$ Others argued that the method is 'especially suited to areas that have both social and clinical dimensions ${ }^{, 6}$ and looked into hypotheses such as 'how qualitative research could answer clinical questions that refer to essentially quantitative data'. ${ }^{3} \mathrm{Hull}^{4}$ emphasized that qualitative research deals with social, as opposed to clinical phenomena, and Poses \& Isen ${ }^{3}$ advised not to use the evidence from qualitative studies until better guidelines for qualitative research become available. The method is also criticized for being subject to researcher bias and for lacking reproducibility and generalizability. ${ }^{7}$ This is because many researchers neglect the importance of giving an adequate description of their theoretical concepts and methods used in their research. $^{7}$

It is evident from the above discussion that there remains controversy about whether qualitative research can address traditionally clinical questions and whether it can generate convincing evidence to improve the quality of care. In contrast, quantitative studies are often criticized as they cannot answer 'appropriate and worthwhile' questions due to their focus on what is measurable, and ultimately may mislead researchers by reducing many dimensions of clinical experience into a single numerical dimension. ${ }^{6}$ Qualitative research can explore complex clinical phenomena which quantitative research cannot and it can reach aspects of complex behaviors, motivations, perceptions, attitudes, and interactions which quantitative methods cannot. ${ }^{8.9}$ Qualitative methods can help bridge the gap between scientific evidence and clinical practice. $^{10}$

Over the past two decades or so, there has been a sustained growth in the use of qualitative methods in medicine and health services research. ${ }^{3,5,7}$ This expansion of qualitative research in healthcare has provided a range of insightful accounts into the factors that influence health and diseases in the community. ${ }^{7}$ The method is useful for answering questions that focus on measuring the extent and range of particular phenomena and helps to understand the phenomena in natural settings by examining the meanings, experiences and views of the participants. Qualitative research uncovers beliefs, values, and motivations that underlie individual health behaviors. ${ }^{11}$ Such research can also examine the organizational culture and factors related to healthcare delivery that influence organizational efficiency and quality of care. ${ }^{12}$ Qualitative studies use inductive (starting with observations and developing hypotheses), rather than deductive (starting with extant hypotheses and testing them with observations) approaches. Qualitative methods should be considered when the research aims to investigate complex phenomena which are difficult to measure quantitatively. ${ }^{13}$ Qualitative methods should not be considered as an 'alternative methodology' of health service research; both methods can indeed be seen as complementary and both are necessary for a comprehensive understanding of a problem and underlying mechanisms. ${ }^{7,13}$ Mixed methods are increasingly recognized as valuable, and are used in larger studies. ${ }^{14}$

Grounded theory, phenomenology and ethnography are three approaches used in qualitative research. Phenomenology and ethnography are more commonly used qualitative approaches in healthcare. ${ }^{7}$ Grounded theory approach is a commonly used method in the social sciences to inductively generate or discover a theory out of the data. ${ }^{7}$ In ethnography, the researcher studies the structure and function of a group of people. ${ }^{7}$ It often relies on participant observation through prolonged field work and may include other qualitative and quantitative methods. ${ }^{15}$ Ethnography is particularly valuable in understanding the influence of social and cultural norms on the effectiveness of health interventions. ${ }^{15}$ Data

Correspondence: Dr. Sayeeda Rahman, Lecturer, Department of Clinical Sciences, School of Medical Sciences, University of Bradford, Bradford BD7 1DP, West Yorkshire, UK. E-mail: srahman6@bradford.ac.uk.

South East Asia Journal of Public Health 2013;3(1):69-71. (C) 2013 Rahman \& Majumder, publisher and licensee Public Health Foundation Bangladesh. This is an Open Access article which permits unrestricted non-commercial use, provided the original work is properly cited. 
collection methods vary in ethnographic studies with observation and interviews being the most popular methods. The use of ethnographic research in medical education has produced a number of insightful accounts into its role, functions and difficulties in the preparation of medical students for clinical practice. ${ }^{16}$ Phenomenology seeks to describe how individuals experience a specific phenomenon and generates a deeper understanding of the 'essence' or meaning of a particular phenomenon from the individual's perspective. ${ }^{17}$

Sampling strategy in qualitative research is more time consuming and expensive and largely determined by the purpose of the study. ${ }^{7}$ In qualitative research, statistical representativeness is not considered a key requirement and is not normally sought. ${ }^{7}$ Qualitative sampling is based on purposeful or theoretical sampling principles to identify relevant participants. ${ }^{8}$ Qualitative research instruments used for data collection include in-depth interviews, focus groups, observation, and document review. Semi-structured tools are more frequently used in healthcare-related qualitative research. ${ }^{7}$ Serial interview studies are widely used by social science; however, they remain underused in medicine. ${ }^{18}$ Another highly popular method is the focus group approach which involves a small interactive group of people discussing and commenting on issues and questions prompted by the researcher. ${ }^{17}$ Qualitative research studies typically generate a substantial amount of data in the form of transcripts and observational field notes. ${ }^{7,18}$ The systematic and rigorous preparation and analysis of this data is time consuming and labor intensive. ${ }^{18}$ Computer packages are available for data analysis and improve the efficiency of data management. ${ }^{7}$

Recently, there has been a greater acceptance of the qualitative approach, even as a stand-alone method, in health care research. ${ }^{7}$ It is also hopeful that institutions that control funding for research have developed ethical guidelines for assessing qualitative studies. ${ }^{22}$ This shows formal acceptance of this method in medicine and healthcare which was previously dominated by quantitative methods. ${ }^{7}$ Qualitative and mixed methods have also been used in a number of areas of clinical medicine and primary care. ${ }^{19-23}$ Quality of healthcare is one of the areas where qualitative methods can be used. ${ }^{7}$ Qualitative research is widely used to study issues related to doctor-patient interaction, especially in general practice. ${ }^{7}$ Research has been conducted to identify cultural and social factors that affect healthcare positively or negatively which was used to improve quality of care. ${ }^{7}$ The use of qualitative research in healthcare enables researchers to answer questions that may not be easily answered by quantitative methods. ${ }^{10}$ However, the introduction of qualitative methods in healthcare needs a thorough understanding of the concepts, theories, methodology and clinical applications. Trained and experienced researchers are needed as good qualitative analysis depends on the skill, vision and integrity of the researcher doing that analysis. ${ }^{18}$ Training healthcare researcher about the criteria for evaluating qualitative research using appropriate theoretical and methodological framework is also crucial. ${ }^{24}$
Historically, health service research in Asian countries does not receive high priority; ${ }^{25,26}$ for example, it has been demonstrated that the average number of research articles published in the region was much less than those of developed countries. ${ }^{27}$ It was also revealed that a higher proportion of qualitative research was associated with journals published in the UK or USA in comparison to other countries. ${ }^{2}$ There is an urgent need to boost the research activities by creating a 'research culture' in healthcare, incorporating research methodology, both quantitative and qualitative, in undergraduate and postgraduate medical curricula. ${ }^{25,28}$ Healthcare service research is crucial for the Asian countries to produce first-hand evidence to identify the extent and burden of health-related problems, identify priority areas, and to formulate a health policy to utilize the scarce resources available in healthcare sector.

\section{References}

1. Dahlberg K. Editorial. Int J Qual Stud Health Well-being 2006; 1: 130-2.

2. Shuval K, Harker K, Roudsari B, Groce NE, Mills B, Siddiqi Z, Shachak A. Is Qualitative Research Second Class Science? A Quantitative Longitudinal Examination of Qualitative Research in Medical Journals. PLoS One 2011;6 (2):e16937.

3. Poses RM, Isen AM. Qualitative Research in Medicine and Health Care: Questions and Controversy. J Gen Intern Med 1998;13(1):32-8.

4. Hull SA. The method of Balint group work and its contribution to research in general practice. Fam Pract 1996;13:S10-2.

5. Helman C. The application of anthropological methods in general practice research. Fam Pract 1996;13:S13-6.

6. Berkwits M, Aronowitz R. Different questions beg different methods. $J$ Gen Intern Med 1995;10:409-10.

7. Al-Busaidi ZQ. Qualitative Research and its Uses in Health Care. Sultan Qaboos U Med J 2008;8(1):11-9.

8. Pope C, Mays N. Reaching the parts other methods cannot reach: an introduction to qualitative methods in health and health services research. BMJ 1995;311:42-5.

9. Gilchrist VJ, Engel JD. Qualitative research and clinical care. J Fam Pract 1995;41:229-30.

10. Green J, Britten N. Qualitative research and evidence based medicine. BMJ 1998;316 (7139):1230-2.

11. Berkwits M, Inui TS. Making use of qualitative research techniques. J Gen Intern Med 1998; 13: 195-199. 
12. Sofaer S, Firminger K. Patient perceptions of the quality of health services. Annu Rev Public Health 2005; 26: 513-59.

13. Curry LA, Nembhard IM, Bradley EH. Qualitative and mixed methods provide unique contributions to outcomes research. Circulation 2009;119 (10):1442-52.

14. Creswell JW, Piano Clark V. Designing and Conducting Mixed Methods Research. Thousand Oaks, Calif: Sage, 2007.

15. Savage J. Ethnography and health care. BMJ 2000; 321: 1400-2.

16. Reeves S, Peller J, Goldman J, Kitto S. Ethnography in qualitative educational research: AMEE Guide No. 80. Med Teach 2013;35(8):e1365-79.

17. Patton MQ. Qualitative Research and Evaluation Methods. 3rd ed. Thousand Oaks, Calif: Sage, 2002.

18. Pope C, Ziebland S, Mays N. Qualitative research in health care. Analysing qualitative data. BMJ 2000;320(7227):114-6.

19. Doering LV, McGuire AW, Rourke D. Recovering from cardiac surgery: what patients want you to know. Am J Crit Care 2002; 11: 333-43.

20. Woodgate R. Part II: a critical review of qualitative research related to children's experiences with cancer. J Pediatr Oncol Nurs 2000; 17: 207 -28 .
21. Creswell JW, Fetters MD, Ivankova NV. Designing a mixed methods study in primary care. Ann Fam Med 2004; 2: 7-12.

22. Twohig PL, Putnam W. Group interviews in primary care research: advancing the state of the art or ritualized research? Fam Pract 2002;19:27884.

23. Verbeek J, Sengers MJ, Riemens L, Haafkens J. Patient expectations of treatment for back pain: a systematic review of qualitative and quantitative studies. Spine 2004; 29: 2309-18.

24. Cohen DJ, Crabtree BF. Evaluative Criteria for Qualitative Research in Health Care: Controversies and Recommendations. Ann Fam Med 2008 Jul-Aug;6(4):331-9.

25. Majumder MAA. Issues and Priorities of Medical Education Research in Asia. Ann Acad Med Singapore 2004;33:257-63.

26. Majumder MAA, Rahman S, Shaban SF, N Rahman, Islam Z. A PubMed-Based Quantitative Analysis of Biomedical Publications in the SAARC Countries: 1985-2009. J Coll Physicians Surg Pak 2012;22:1-7.

27. Rahman M, Fukui T. Biomedical publication Global profile and trend. Public Health 2003;117:274-80.

28. Rahman S, Majumder MAA, Shaban SF, N Rahman, M Ahmed. Physician Participation in Clinical Research and Trials: Issues and Approaches. Adv Med Educ Pract 2011,2:1-9. 